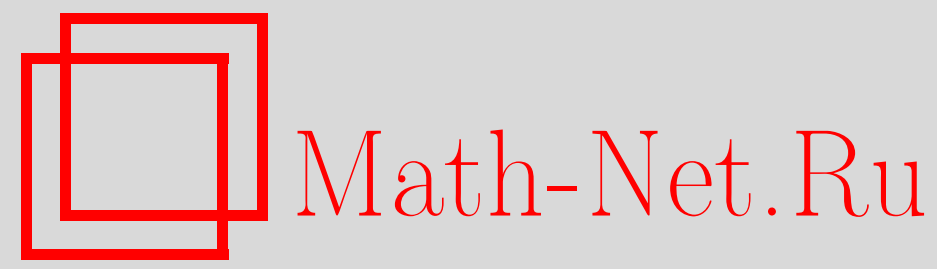

Ф. С. Насыров, О локальных временах для функций и случайных процессов. II, Теория вероятн. и ее примен., 1996, том 41, выпуск 2, 284-299

DOI: https://doi.org/10.4213/tvp2933

Использование Общероссийского математического портала Math-Net.Ru подразумевает, что вы прочитали и согласны с пользовательским соглашением http://www.mathnet.ru/rus/agreement

Параметры загрузки:

IP : 34.227 .88 .159

26 апреля 2023 г., 12:03:43

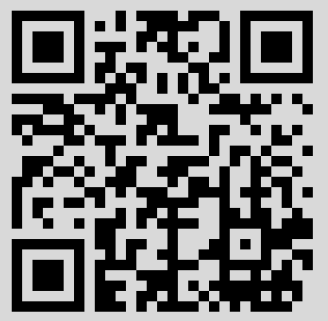




\section{О ЛОКАЛЬНЫХ ВРЕМЕНАХ ДЛЯ ФУНКЦИЙ И СЛУЧАЙНЫХ ПРОЩЕССОВ. II}

Пусть $X(t), 0 \leqslant t \leqslant 1$, - вещественнозначная измеримая функция, обладающая локальным временем $\alpha(t, u), 0 \leqslant t \leqslant 1, u \in \mathbf{R}$. Если последнее непрерывно по $t$ при п.в. $u$, то распределение $F(t, x)=$ $\int_{\mathbf{R}} \mathbb{I}\{\alpha(t, u)>x\} d u$ и монотонная перестройка $\alpha^{*}(t, u)=\inf \{x:$ $F(t, x)<u\}$ локального времени $\alpha(t, u)$ являются локальньми временами для $\xi(s)=\alpha(s, X(s))$ и $\xi^{*}(s)=F(s, \xi(s)), 0 \leqslant s \leqslant 1$, соответственно.

Ключевые слова и фразы: локальное время, распределение и монотонная перестройка функции, ортогональное разложение, броуновское движение.

Настоящая работа является продолжением статьи [5]; нумерация формул и утверждений продолжает нумерацию первой статьи.

$\S 4$. Ортогональное разложение процесса $\xi(s)$. Уравнение для совместного распределения $\mathbf{P}\{\xi(t)<x, X(t)<y\}$

До сих пор мы придерживались точки зрения теории функций, рассматривая $X(s)$ как (неслучайную) функцию. В дальнейшем всюду, если не оговорено противное, мы будем предполагать, что $X(s), s \in[0,1],-$ вешественнозначный стохастически непрерывный случайный процесс с локальным временем $\alpha(t, u)$, с вероятностью 1 непрерывным по $t$ при II.B. $u$.

В частности (см. [13]), если $X(s), s \in[0,1]$, - пентрированный гауссовский процесс с измеримыми траекториями и с $\sigma^{2}(s, t)=\mathbf{E}(X(s)-$ $X(t))^{2}$, удовлетворяюшим условию $\int_{0}^{1} \sigma^{-1}(s, t) d s<\infty$ при п.в. $t$, то все указанные выше требования выполнены. Другие условия непрерывности локальных времен, отвечающих различным классам случайных процессов, обсуждаются в работах [7]-[9], [11]-[13].

Переходя от отдельных траекторий процесса к самому процессу, мы можем перенести полученные выше результаты на случайные процессы; формально это можно сделать, например, в следующей форме:

Теорема 5. Если прочесс $X(s)$ обладает локальным временем $\alpha(t, u), t \in[0,1], u \in \mathbf{R}$, с вероятностью 1 непрерывным по $t$ при п.в. $u$,

*Уфимский авиационный технический университет, кафедра математики, К. Маркса, 12, 450025 Уфа, Россия. 
то для почти всех траекторий прочесса $X(s)$ справедливь доказанные выне в 22,3 утверждения.

Ниже в этом параграфе, во-первых, следуя подходу, принятому в работах [10], [4], мы получим ортогональное разложение локального времени вдоль траектории $\xi(t)$, и, во-вторых, выведем интегральное уравнение для совместного распределения $\mathbf{P}\{\xi(t) \leqslant x, X(t) \leqslant y\}$.

Для того, чтобы перейти к первому вопросу, приведем для удобства необходимые в дальнейшем сведения. Пусть $X(s), s \in[0,1],-$ случайный процесс с двумерной плотностью распределения $p(x, y, s, \tau), x, y \in \mathbf{R}$, $s, \tau \in[0,1]$, и одномерной плотностью $p(x)$, не зависящей от $s$.

Процесс $X(s)$ назовем процессом равномерного PB-muna (positive bivariate type), если существует $\delta, 0<\delta \leqslant 1$, такое, что при всех $s, \tau \in$ $[0,1], s \neq \tau,|\tau-s|<\delta$, выполняются условия:

(A) Функции

$$
\frac{p(x, y, s, \tau)}{p(x) p(y)}
$$

принадлежат произведению пространств $H=L_{2}(p(x) d x) \times L_{2}(p(y) d y)$, где $L_{2}(p(x) d x)=\left\{f(x), x \in \mathbf{R}: \int_{\mathbf{R}} f^{2}(x) p(x) d x<\infty\right\}$.

(Б) Ядра (21) раскладываются в ряд

$$
\frac{p(x, y, s, \tau)}{p(x) p(y)}=\sum_{n=0}^{\infty} r_{n}(s, \tau) e_{n}(x) e_{n}(y),
$$

сходяшийся в смысле сходимости в $H$, где $\left\{e_{n}(x)\right\}_{n=0}^{\infty}-$ нормированная последовательность собственных функций, не зависяших от $s$ и $\tau$, a $\left\{r_{n}(s, \tau)\right\}_{n=0}^{\infty}$ - последовательность собственных значений линейного оператора, порожденного в $H$ ядром (21), причем $r_{0}=1, e_{0}=1$, $r_{n}(s, \tau) \geqslant 0, n \in \mathbf{N}$.

Будем говорить, что процесс $Y(s), s \in[0,1]$, принадлежит почтu равномерному РВ-типу, если существует измеримая положительная функция $b(s), s \in[0,1]$, такая, что процесс $X(s)=(b(s))^{-1} Y(s), s \in[0,1]$, принадлежит равномерному РВ-типу. Ниже, если это специально не оговорено, фиксируется случайный процесс только что описанного вида, а для всех объектов, связанных с процессом $X(s)$, будут использованы обозначения этого раздела. В дальнейшем предполагается, что при п.в. $s$ и $\tau$ функции $p(x, y, s, \tau)$ непрерывны по $(x, y)$ на $\mathbf{R}^{2} ;$ функция $p(x)$ непрерывна на $\mathbf{R}$.

Для удобства приведем основной результат работы [4], который нам понадобится ниже.

Теорема 6. Предположим, ито выполнены следующие условия:

$$
\begin{aligned}
\text { 1. } \int_{0}^{1} \int_{0}^{1} \int_{\mathbf{R}}\left[p\left(\frac{x}{b(s)}, \frac{x}{b(s)}, s, \tau\right) p\left(\frac{x}{b(\tau)}, \frac{x}{b(\tau)}, s, \tau\right)\right]^{1 / 2} d x \\
\quad \times \frac{d s d \tau}{b(s) b(\tau)}<\infty
\end{aligned}
$$


2. При всех $x \in \mathbf{R}, n \in \mathbf{N}$

$$
\int_{0}^{1} p\left(\frac{x}{b(s)}\right)\left|e_{n}\left(\frac{x}{b(s)}\right)\right| \frac{d s}{b(s)}<\infty
$$

3. Интеграл

$$
\varphi(x, y)=\int_{0}^{1} \int_{0}^{1} p\left(\frac{x}{b(s)}, \frac{x}{b(\tau)}, s, \tau\right) \frac{d s d \tau}{b(s) b(\tau)}
$$

комечен и является непрерывной по $(x, y) \in \mathbf{R}^{2}$ функцией;

4. При п.в. $s$ u $\tau, n \in \mathbf{N}$

$$
\int_{\mathbf{R}}[p(x, x, s, \tau)]^{1 / 2}\left|e_{n}(x)\right| d x<\infty
$$

5. Система функиий $\left\{e_{n}(x)\right\}_{n=0}^{\infty}$ полна в $L_{2}(p(x) d x)$.

Тогда при каждом $T \in \mathcal{B}$

(a) $P$ я $\partial$

$$
\widetilde{\alpha}(T, x)=\sum_{n=0}^{\infty} g_{n}(T, x)
$$

при любом $x$ сходится в среднем көадратическом; при этом случайнье величины

$$
g_{n}(T, x)=\int_{T} p\left(\frac{x}{b(s)}\right) e_{n}\left(\frac{x}{b(s)}\right) e_{n}\left(\frac{Y(s)}{b(s)}\right) \frac{d s}{b(s)}
$$

отвечающие разным значениям $n$, попарно ортогональны;

(б) Случайный прочесс $\widetilde{\alpha}(T, x), x \in \mathbf{R}$, непрерывен в среднем көадратическом;

(в) Существует локальное время $\alpha(T, x)$ для прочесса $Y(s)$ и с вероятностью 1 при п.в. $x \quad \alpha(T, x)=\widetilde{\alpha}(T, x)$, то есть $\widetilde{\alpha}(T, x)$ является версией локального времени для прочесса $Y(s)$.

Пусть $X(s), s \in[0,1]$, - случайный пропесс (не обязательно почти равномерного РВ-типа) с локальным временем $\alpha(t, x)$, непрерывным по $t$ с вероятностью 1 при п.в. $x$. Положим $X^{\tau}(s)=X(\tau)-X(s), s \in[0,1]$ (все характеристики случайного процесса $X^{\tau}(s)$ будем обозначать так же, как и в случае пропесса $X(s)$, но с добавлением индекса $\tau$ навер$\mathrm{xy,} \mathrm{например,} p^{\tau}(x)$ - одномерная плотность процесса $X^{\tau}(s)$, поскольку $p(x)$ - соответствуюшая плотность пропесса $X(s))$.

Теорема 7. Iредположим, что при п.в. $\tau$ выполнены следующие условия:

1. Случайный прочесс $X^{\tau}(s)=X(\tau)-X(s), s \in[0,1]$, принадлежит почти равномерному РB-muпу;

2. Дая прочесса $X^{\tau}(s)$ выполнены предположения 1-5 теоремы 6. Tozda 
(a) $P g \partial$

$$
\tilde{\xi}(\tau)=\sum_{n=0}^{\infty} p^{\tau}(0) e_{n}^{\tau}(0) \int_{0}^{\tau} e_{n}^{\tau}\left(\frac{X(\tau)-X(s)}{b^{\tau}(s)}\right) \frac{d s}{b^{\tau}(s)}
$$

при п.в. $\tau$ сходится в среднем квадратическом; при этом случайные величины

$$
\int_{0}^{\tau} e_{n}^{\tau}\left(\frac{X(\tau)-X(s)}{b^{\tau}(s)}\right) \frac{d s}{b^{\tau}(s)}
$$

отвечающие разным значениям $n$, попарно ортогональны; $\tilde{\xi}(\tau)$.

(б) С вероятностью 1 при п.в. $\tau$ выполндется равенство $\xi(\tau)=$

Д ок а $з$ а т е л ь с в о. Зафиксируем любое $\tau \in[0,1]$, для которого справедливы предположения 1 и 2 теоремы 7. В силу теоремы 6 , опираясь на тождество (1), выводим, что при п.в. $\omega$ локальные времена для процессов $X^{\tau}(s)$ и $X(s)$ связаны между собой следуюшим образом: для любого $t \in[0,1] \alpha^{\tau}(t, y)=\alpha(t, X(\tau)+y)$ при п.в. $y$. Ввиду теоремы о дифференцировании интегралов, дополнение множества.

$$
\Gamma_{t, \omega}=\left\{z \in \mathbf{R}: \lim _{\varepsilon \downarrow 0} \frac{1}{2 \varepsilon} \int_{\{y:|y|<\varepsilon\}} \alpha(t, y+z) d y=\alpha(t, z)\right\}
$$

есть множество нулевой лебеговой меры. Значит в силу существования локального времени для процесса $X(s)$

$$
\int_{0}^{1} \mathbb{I}\left\{X(\tau) \in \mathbf{R} \backslash \Gamma_{t, \omega}\right\} d \tau=0 \quad \text { п.н. }
$$

а это означает, что при каждом $t \in[0,1]$ п.н.

$$
\lim _{\varepsilon \downarrow 0} \frac{1}{2 \varepsilon} \int_{\{y:|y|<\varepsilon\}} \alpha^{\tau}(t, y) d y=\alpha(t, X(\tau)) \quad \text { при п.в. } \quad \tau .
$$

Взяв счетное встоду плотное в $[0,1]$ множество $\left\{t_{k}\right\}_{k=1}^{\infty}$, в силу монотонности и непрерывности $\alpha(t, x)$, выводим, что соотношение (25) справедливо одновременно для всех $t$ при п.в. $\tau$ п.н. Отсюда, в частности, следует, что при п.в. $\tau$ п.н.

$$
\lim _{\varepsilon \downarrow 0} \frac{1}{2 \varepsilon} \int_{\{y:|y|<\varepsilon\}} \alpha^{\tau}(\tau, y) d y=\xi(\tau)
$$

Поскольку, в силу неравенства Йенсена, для любого $t$

$$
\begin{aligned}
& \mathbf{E}\left(\frac{1}{2 \varepsilon} \int_{\{y:|y|<\varepsilon\}}\left[\alpha^{\tau}(t, y)-\alpha^{\tau}(t, 0)\right] d y\right)^{2} \\
& \quad \leqslant \frac{1}{2 \varepsilon} \int_{\{y:|y|<\varepsilon\}} \mathbf{E}\left[\alpha^{\tau}(t, y)-\alpha^{\tau}(t, 0)\right]^{2} d y \quad \text { при п.в. } \tau,
\end{aligned}
$$


то для тех же $\tau$, в силу непрерывности в среднем квадратическом функции $\alpha^{\tau}(\tau, y)$ по переменной $y$,

$$
\lim _{\varepsilon \downarrow 0} \mathbf{E}\left(\frac{1}{2 \varepsilon} \int_{\{y:|y|<\varepsilon\}} \alpha^{\tau}(\tau, y) d y-\alpha^{\tau}(\tau, 0)\right)^{2}=0
$$

Это означает, что $\alpha^{\tau}(\tau, 0)=\xi(\tau)$ п.н. при п.в. $\tau$. Но при п.в. $\tau$ для локального времени $\alpha^{\tau}(\tau, 0)$ справедливо ортогональное разложение (23); осталось доказать, что п.н. при п.в. $\tau \xi(\tau)=\alpha^{\tau}(\tau, 0)$. Последнее вытекает из равенства

$$
\mathbf{E}\left(\int_{0}^{1}\left(\xi(\tau)-\alpha^{\tau}(\tau, 0)\right)^{2} d \tau\right)=\int_{0}^{1} \mathbf{E}\left(\xi(\tau)-\alpha^{\tau}(\tau, 0)\right)^{2} d \tau=0
$$

П р и м е р 2. Пусть $Y(s), s \in[0,1]$ - сепарабельный гауссовский процесс с нулевым средним и ковариацией $B(s, \tau)=\mathbf{E}(Y(s) Y(\tau))$. Обозначим через $p(x, y, s, \tau), x, y \in \mathbf{R}, s, \tau \in[0,1]$, двумерные плотности распределения процесса $X(s)=(b(s))^{-1} Y(s)$, где $b(s)=(B(s, s))^{1 / 2}$. Предположим, что выполнены условия: $B(s, \tau) \geqslant 0$ для всех $s, \tau \in[0,1]$; при всех $s, \tau \in[0,1], s \neq \tau, B(s, \tau)[b(s) b(\tau)]^{-1} \neq 1$. Тогда (см. [10]) процесс $X(s), s \in[0,1]$, является процессом равномерного РВ-типа и для функций $p(x, y, s, \tau)$ при любых $s, \tau \in[0,1]$ справедливо разложение $(22)$ с $p(x)=(1 / \sqrt{2} \pi) \exp \left\{-x^{2} / 2\right\}, r_{n}(s, \tau)=[B(s, \tau) /(b(s) b(\tau))]^{n}$, $e_{n}(x)=(1 / \sqrt{n !}) H_{n}(x)$, где $H_{n}(x), x \in \mathbf{R}, n \in \mathbf{N}$, - классические многочлены Эрмита, связанные с весовой функцией $\exp \left\{-x^{2} / 2\right\}$. Следовательно процесс $Y(s), s \in[0,1]$, с ковариацией, удовлетворяющей перечисленным выше условиям, будет процессом почти равномерного РВ-типа.

Если

$$
\int_{0}^{1} \int_{0}^{1} \frac{d s d \tau}{\sqrt{B(s, s) B(\tau, \tau)-B^{2}(s, \tau)}}<\infty
$$

то выполнены все предположения теоремы 6 .

Положим $\sigma\left(\tau, s, s^{\prime}\right)=\mathbf{E}\left[(Y(\tau)-Y(s))\left(Y(\tau)-Y\left(s^{\prime}\right)\right)\right]$. Если при п.в. $\tau$ выполняются условия:

1. $\sigma\left(\tau, s, s^{\prime}\right) \geqslant 0$ при любых $\tau, s, s^{\prime}$;

2.

$$
\int_{0}^{1} \int_{0}^{1} \frac{d s d s^{\prime}}{\sqrt{\sigma(\tau, s, s) \sigma\left(\tau, s^{\prime} s^{\prime}\right)-\sigma^{2}\left(\tau, s, s^{\prime}\right)}}<\infty
$$

то все предположения теоремы 7 выполнены.

Заметим, что определение локального времени и связанные с ним конструкции очевидным образом переносятся с параметрического множества $[0,1]$ на $[0,+\infty)$.

Теорема 8. Пусть $X(s), s \in[0,+\infty),-$ вещественнозначный стохастически жепрерывный случайный прочесс с независимыми приращениями, обладающий локальным временем $\alpha(t, u), t \in[0,+\infty), u \in \mathbf{R}$, с вероятностью 1 непрерывным по переменньм $(t, u)$. 
Тогда функиия

$$
u(t, x, y)=\mathbf{P}\left\{\int_{0}^{t} \psi(s) \alpha(d s, X(t)) \leqslant x, X(t) \leqslant y\right\} \psi(t),
$$

где $\psi(\cdot)$ - непрерывная неотричательная функиия, удовлетворяет в области $t \geqslant 0, x \geqslant 0, y \in \mathbf{R}$ уравнению

$$
\begin{aligned}
x \psi(t) \mathbf{P}\{X(t) \leqslant y\}-\int_{0}^{x} u(t, z, y) d z \\
\quad=\psi(t) \lim _{\varepsilon \downarrow 0} \frac{1}{2 \varepsilon} \int_{0}^{t} u(s, x, y) \mathbf{P}\{|X(t)-X(s)|<\varepsilon\} d s .
\end{aligned}
$$

Д о к а з а т е л ь с т в о. Временно зафиксируем отдельную траекторию процесса $X(s)$, обладающую локальным временем $\alpha(t, u)$, непрерывным по переменным $(t, u)$. Обозначим

$$
\alpha_{\psi}(t, u)=\int_{0}^{t} \psi(s) \alpha(d s, u), \quad \xi_{\psi}(t)=\alpha_{\psi}(t, X(t)) .
$$

Заметим, что, в силу теоремы Хелли, функция $\alpha_{\psi}(t, u)$ непрерывна по $u$. Для любого $x>0$ ввиду леммы 1 получим

$$
\min \left(x, \alpha_{\psi}(t, u)\right)=\int_{0}^{t} \mathbb{I}\left\{\alpha_{\psi}(s, u)<x\right\} \alpha_{\psi}(d s, u) .
$$

Умножим обе части тождества $(27)$ на $\mathbb{I}\{u \leqslant y\}$. Поскольку правая часть полученного тождества непрерывна по $u$ за исключением точки $u=y$, то для $u \neq y$ ее можно переписать в виде

$$
\lim _{\varepsilon \downarrow 0} \frac{1}{2 \varepsilon} \int_{\mathbf{R}} \mathbb{I}\{|u-v|<\varepsilon, v \leqslant y\} \int_{0}^{t} \mathbb{I}\left\{\alpha_{\psi}(s, v)<x\right\} \psi(s) \alpha_{\psi}(d s, v) d v .
$$

В силу формулы (1) мы приходим к тождеству

$$
\begin{array}{rl}
\int_{0}^{x} & \mathbb{I}\left\{z \leqslant \alpha_{\psi}(t, u)\right\} d z \cdot \mathbb{I}\{u \leqslant y\} \\
\quad=\lim _{\varepsilon \downarrow 0} \frac{1}{2 \varepsilon} \int_{0}^{t} \mathbb{I}\left\{|u-X(s)|<\varepsilon, X(s) \leqslant y, \xi_{\psi}(s) \leqslant x\right\} \psi(s) d s .
\end{array}
$$

Положив $u=X(t)$ и учитывая, что $\mathrm{P}\{X(t)=y\}=0$, возьмем от обеих частей тождества (28) математическое ожидание. Поскольку обе части тождества (28) не превосходят $x$, то в силу теоремы Лебега о предельном переходе под знаком интеграла, имеем:

$$
\begin{aligned}
& x \psi(t) \mathbf{P}\{X(t) \leqslant y\}-\int_{0}^{x} u(t, z, y) d z \\
& =\psi(t) \lim _{\varepsilon \downarrow 0} \frac{1}{2 \varepsilon} \int_{0}^{t} \mathbf{P}\left\{|X(t)-X(s)|<\varepsilon, \xi_{\psi}(s) \leqslant x, X(s) \leqslant y\right\} \psi(s) d s .
\end{aligned}
$$


Используя затем тот факт, что $X(s)$ - процесс с независимыми приращениями, приходим к уравнению (26).

3 а м е ч а н и е. Предположим дополнительно, что $X(s), s>0-$ однородный процесс с независимыми приращениями. Пусть функция $\psi(s)=1$ для всех $s \in[0,1]$, тогда уравнение (26) сводится с помощью преобразования Лапласа к дифференциальному уравнению 1-го порядка. Действительно, положим

$$
\begin{aligned}
U(\lambda, x, y) & =\int_{0}^{+\infty} e^{-\lambda t} u(t, x, y) d t \\
W(\lambda) & =\lim _{\varepsilon \downarrow 0} \frac{1}{2 \varepsilon} \int_{0}^{+\infty} e^{-\lambda t} \mathbf{P}\{|X(t)|<\varepsilon\} d t, \\
V(\lambda, y) & =\int_{0}^{+\infty} e^{-\lambda t} \mathbf{P}\{X(t) \leqslant y\} d t .
\end{aligned}
$$

Поскольку интеграл в правой части уравнения (26) при $\psi(t)=1$ представляет собой свертку двух функций, то применение преобразования Лапласа перевоцит уравнение (26) в уравнение

$$
V(\lambda, y)-U(\lambda, x, y)=W(\lambda) \frac{\partial}{\partial x} U(\lambda, x, y) .
$$

Решая данное уравнение с учетом начального условия $V(\lambda, 0, y)=0$, получаем

$$
U(\lambda, x, y)=V(\lambda, y)\left(1-e^{x / W(\lambda)}\right) .
$$

В случае процесса стандартного броуновского движения $X(s)$ из равенства (29) выводим равенство $\mathbf{P}\{\xi(t) \leqslant x\}=\mathbf{P}\{|X(t)| \leqslant x\}$.

\section{§5. Броуновское локальное время}

Пусть $X(s), s \in[0,1],-$ стандартное броуновское движение с локальным временем $\alpha(t, u), t \in[0,1], u \in \mathbf{R}$, непрерывным с вероятностью 1 по переменным $(t, u)$.

Найдем совместное распределение процесса $X(s)$ и локального времени вдоль траектории $\xi(s)$. Заметим, что для некоторых процессов, в том числе и для процесса броуновского движения, известны распределения $\mathbf{P}\{\alpha(t, u) \leqslant x\}$. Поэтому, предполагая, что в тождестве (6) $X(s)$ и $\xi(s)$ - случайные процессы, и взяв от обеих частей данного тождества математическое ожидание, получим

$$
\int_{0}^{t} \mathbf{P}\{\xi(s) \leqslant x, X(s) \leqslant y\} d s=\int_{0}^{x} \int_{-\infty}^{y} \mathbf{P}\{\alpha(t, u)>z\} d u d z .
$$

Точно так же, опираясь, например, хотя бы на второе утверждение предложения 3 , выводим

$$
\int_{0}^{t} \mathbf{P}\{\xi(s)-f(X(s)) \leqslant y, X(s) \leqslant x\} d s
$$




$$
=\int_{-\infty}^{y} \int_{-\infty}^{x} \mathbf{P}\{\alpha(t, u)>z+f(u)\} \mathbb{I}\{f(u)>-z\} d u d z
$$

Из равенства (30) следует, что

$$
\mathbf{P}\{\xi(t) \leqslant x, X(t) \leqslant y\}=\frac{\partial}{\partial t} \int_{0}^{x} \int_{-\infty}^{y} \mathbf{P}\{\alpha(t, u)>z\} d u d z
$$

Аналогичное соотношение вытекает из равенства (31). В частности, известно (см. $[3$, с. 383$])$, что для броуновского движения $X(s)$

$$
\mathbf{P}\{\alpha(t, u)>z\}=\frac{2}{\sqrt{\pi}} \int_{(z+|u|) /(\sqrt{2} t)}^{+\infty} e^{-v^{2}} d t, \quad z>0
$$

Поэтому

$$
\mathbf{P}\{\xi(t) \leqslant x, X(t) \leqslant y\}=\frac{1}{\sqrt{2 \pi t}} \int_{-\infty}^{y}\left[e^{-u^{2} /(2 t)}-e^{-(x-|u|)^{2} /(2 t)}\right] d u .
$$

Как отмечено в работе [1], в число применений броуновского локального времени входят различные варианты обобщенных формул Ито, а первым результатом в этом направлении является формула Танаки: с вероятностью 1 для всех $(\tau, u)$

$$
\frac{1}{2} \alpha(\tau, u)=(X(\tau)-u)^{+}-(-u)^{+}-\int_{0}^{\tau} \mathbb{I}\{X(s)>u\} d X(s)
$$

где $(z)^{+}=\max (z, 0)$. Мы также докажем одно подобное утверждение. Для этого нам потребуется следующий результат.

Лемма 2. Пусть $y(s), s \in[0,1],-$ непрерывная функиия $c$ локальным временем $\alpha(t, u), t \in[0,1], u \in \mathbf{R}$, непрерывным по переменным $(t, u)$. Тогда для любых $u \in \mathbf{R}, x>0, t \in[0,1]$

$$
\begin{aligned}
& {\left[y\left(\alpha_{u, t}^{-1}(x)\right)-u\right] \mathbb{I}\{\alpha(t, u)>x\}=0,} \\
& {\left[\xi\left(\alpha_{u, t}^{-1}(x)\right)-x\right] \mathbb{I}\{\alpha(t, u)>x\}=0,}
\end{aligned}
$$

где $\alpha_{u, t}^{-1}(x)=\min \{s \leqslant t: \alpha(s, u)=x\}$ - обратная $\kappa \alpha(t, u)$ функция, которая определяется на множестве $x \in(0, \alpha(t, u))$.

Д ок а з а т е л ь с т в о. Заметим, что если $\alpha(t, u)>x>0$, то $u \in\left[\min _{0 \leqslant s \leqslant t} y(s), \max _{0 \leqslant s \leqslant t} y(s)\right]$. Зафиксируем $t \in[0,1], u \in \mathbf{R}, x>0$. Положим $s^{*}=\alpha_{u, t}^{-1}(x)$. В силу построения точки $s^{*}$ и того факта, что носитель меры $\alpha(d s, u)$, порожденной неубывающей функцией $\alpha(s, u), s \in$ $[0,1]$, содержится в множестве $\{s \in[0,1]: X(s)=u\}$, имеем:

$$
\int_{s^{*}-\varepsilon}^{s^{*}} \mathbb{I}\{X(s)=u\} \alpha(d s, u)>0, \quad \alpha\left(s^{*}, u\right)=x
$$


для любого $\varepsilon>0$. Значит, либо $X\left(s^{*}\right)=u$, либо существует последовательность $s_{k} \uparrow s^{*}(k \rightarrow \infty)$ такая, что $X\left(s_{k}\right)=u$. Но, ввиду непрерывности функции $X(s), u=\lim _{k \rightarrow \infty} X\left(s_{k}\right)=X\left(s^{*}\right)$, и, таким образом, первое утверждение леммы 2 доказано. Далее, $\xi\left(s^{*}\right)=\alpha\left(s^{*}, X\left(s^{*}\right)\right)=\alpha\left(s^{*}, u\right)=$ $x$, что завершает доказательство леммы 2.

Положим $J(t, x, u)=\int_{0}^{t} \mathbb{I}\{X(s)>u, \alpha(s, u) \leqslant x\} d X(s)$.

Теорема 9 (обобщенная формула Танаки). Для любых $t \in[0,1]$, $x>0, u \in \mathbf{R}$ с вероятностью 1

$$
\frac{1}{2} \min (x, \alpha(t, u))=\mathbb{I}\{\alpha(t, u) \leqslant x\}(X(t)-u)^{+}-(-u)^{+}-J(t, x, u) .
$$

Д о к аз а те л ь с т в о. Зафиксируем $t \in[0,1], x>0, u \in \mathbf{R}$. Умножим обе части формулы Танаки (32) на $\mathbb{I}\{\alpha(t, u)>x\}$ и положим $\tau$ равным марковскому моменту $\alpha_{u, t}^{-1}(x)=\min \{s \leqslant t: \alpha(s, u)=x\}$. В силу построения марковского момента и формулы (33) получим

$$
\begin{aligned}
. \frac{x}{2} \mathbb{I}\{\alpha(t, u)>x\}=[- & \left.(-u)^{+}-\int_{0}^{\alpha_{u, z}^{-1}(x)} \mathbb{I}\{X(s)>u\} d X(s)\right] \\
& \times \mathbb{I}\{\alpha(t, u)>x\}
\end{aligned}
$$

Ввиду свойств стохастического интеграла, расположенный в правой части равенства стохастический интеграл равен $\int_{0}^{t} \mathbb{I}\{\alpha(s, u) \leqslant x$, $X(s)>u\} d X(s)$. Положим теперь в формуле Танаки (32) $\tau=t$ и умножим обе части полученного равенства на $\mathbb{I}\{\alpha(t, u) \leqslant x\}$.

Заметим, что почти наверное

$$
\mathbb{I}\{\alpha(t, u) \leqslant x\} \int_{0}^{t} \mathbb{I}\{X(s)>u\} d X(s)=\mathbb{I}\{\alpha(t, u) \leqslant x\} J(t, x, u) .
$$

Складывая полученное равенство с равенством (35), в силу тождества $\min (x, y)=x \mathbb{I}\{x<y\}+y \mathbb{I}\{x \geqslant y\}$ получим формулу (34).

3 а м е ч а н и е. В силу равенства (34) стохастический интеграл $J(t, x, u)$ обладает версией, представляющей собой при фиксированных $t, u, \omega$ функцию ограниченной вариации по переменной $x$, поскольку $J(t, x, u)$ есть разность неубывагших функций $\frac{1}{2} \min (x, \alpha(t, u))$ и $\mathbb{I}\{\alpha(t, u) \leqslant x\}(X(t)-u)^{+}$.

Более того, ввиду предположения, что $\alpha(t, u)$ - вариант локального времени, совместно непрерывный по $(t, u)$, из соотношения (34) вытекает, что существует версия $J(t, x, u)$, измеримая относительно переменных $(t, x, u, \omega)$.

Следствие 1. Для любых $t \geqslant 0, x>0, u \in \mathbf{R}$ справедливо равенcm6o

$$
\left(J(t, x, u)-\frac{x}{2}-(-u)^{+}\right) \mathbb{I}\{\alpha(t, u)>x\}=0 .
$$

Соотношение (36) сразу вытекает из равенства (34). 
Следствие 2. Пусть $J(t, x, u)$ - версия стохастического интеграла, измеримая относительно переменных $(t, x, u, \omega)$.

Тогда равенство (34) справедливо п.н. при п.в. $(x, u)$.

Действительно, умножая обе части равенства (34) на $\mathbb{I}(A), A \in \mathcal{F}$, и учитывая, что обе части равенства не превосходят $x$, возьмем от обеих частей равенства (34) математическое ожидание, а затем проинтегрируем по переменным $(x, u) \in\left[x_{1}, x_{2}\right] \times\left[u_{1}, u_{2}\right]$, где $0 \leqslant x_{1}<x_{2}, u_{1}<u_{2}$ произвольные числа. Ввиду произвольности множеств $A,\left[x_{1}, x_{2}\right],\left[u_{1}, u_{2}\right]$ получим требуемое, применив теорему Фубини.

Следствие 3. При любых $t>0, v \in \mathbf{R}, z \geqslant 0$ с веровтностью 1

$$
\begin{aligned}
\frac{1}{2} & \int_{0}^{t} \int_{\xi(s)}^{z} f(x, X(s)) d x \mathbb{I}\{X(s) \leqslant v\} d s \\
= & \int_{-\infty}^{v} \int_{0}^{z} f(x, u)\left[(X(t)-u)^{+}-(-u)^{+}\right] \mathbb{I}\{\alpha(t, u) \leqslant x\} d x d u \\
& -\int_{0}^{t}\left[\int_{-\infty}^{v} \int_{0}^{z} f(x, u) \mathbb{I}\{X(s)>u, \alpha(s, u) \leqslant x\} d x d u\right] d X(s),
\end{aligned}
$$

где $f(\cdot, \cdot)$ - ограниченная (неслучайная) функчия.

Действительно, ввиду следствия 2 к теореме 9, умножим обе части равенства (34) на функцию $f(x, u)$, интегрируем полученное тождество по переменным $(x, u) \in[0, z] \times(-\infty, v]$ и, применив к правой части полученного тождества «стохастическую теорему Фубини» $([2$, с. 123]), получим, что правая часть этого тождества совпадает с выражением, стоящим в правой части равенства (37).

Согласно лемме 1

$$
\min (x, \alpha(t, u))=\int_{0}^{t} \mathbb{I}\{\alpha(s, u) \leqslant x\} \alpha(d s, u),
$$

поэтому левая часть полученного тождества примет вид

$$
\frac{1}{2} \int_{0}^{z} \int_{-\infty}^{v} \int_{0}^{t} \mathbb{I}\{\alpha(s, u) \leqslant x\} \alpha(d s, u) f(x, u) d u d x .
$$

В силу формулы (1) и теоремы Фубини последнее выражение равно левой части соотношения (37).

3 а м е ч а н и е. Другие варианты следствий из формулы (34) можно получить, умножая равенство (34) на неслучайную функцию одной переменной $(f(u)$ или $f(x))$ и интегрируя это тождество только по этой переменной.

Найдем представления некоторых рассматриваемых в данной работе функционалов в виде сумм, содержащих стохастические интегралы. Так, интегрируя тождество (34) по переменной $u \in U, m(U)<\infty$, в силу тождества (2) и упомянутой выше «стохастической теоремы Фубини» 
для времени пребывания $\mu_{t, U}(x)$ процесса $\xi(\tau)$, выводим: с вероятностью 1

$$
\begin{aligned}
\frac{1}{2} \mu_{t, U}(x)= & \int_{U} \mathbb{I}\{\alpha(t, u)<x\}(X(t)-u)^{+} d u-\int_{U}(-u)^{+} d u \\
& -\int_{0}^{t}\left[\int_{U} \mathbb{I}\{\alpha(s, u)<x, X(s)>u\} d u\right] d X(s)
\end{aligned}
$$

Далее, в силу соотношений (7) и (38), для $U \in \mathcal{B}, m(U)<\infty$,

$$
\begin{aligned}
\frac{x}{2} F_{U}(t, x)= & -\int_{U} \mathbb{I}\{\alpha(t, u)<x\}(X(t)-u)^{+} d u \\
& -\int_{U}(-u)^{+} d u-\int_{0}^{t}\left[\int_{U} \mathbb{I}\{\alpha(s, u)<x, X(s)>u\} d u\right] d X(s) \\
& -\frac{1}{2} \int_{0}^{t} \mathbb{I}\{\alpha(t, X(s)) \leqslant x, X(s) \in U\} d s \quad \text { п.н. }
\end{aligned}
$$

Напомним, что если $Z(t), t \geqslant 0$, - пропесс броуновского движения, $Z(0)=0$, то согласно уравнению Скорохода $([2$, с. 127])

$$
|Z(t)|=B(t)+\gamma(t, 0) \quad \text { п.н., }
$$

где $B(t)$ - новое броуновское движение, $B(t)=\int_{0}^{t} \operatorname{sgn}(Z(s)) d Z(s)$, $\gamma(t, 0)$ - локальное время процесса $Z(s)$ в нуле, при этом П.н. $\gamma(t, 0)=$ - $\min _{0 \leqslant s \leqslant t} B(s)$. Формула (34) позволяет получить одно обобщение уравнения (39).

Теорема 10 (обобшенное уравнение Скорохода). Пусть $\Phi(z)$, $z \geqslant 0$, - абсолютно непрерывная функиия,

$$
\Phi(z)=\int_{z}^{\infty} \psi(y) d y, \quad \psi(y)>0, \quad y \in[0,+\infty)
$$

Тогда с вероятностью 1

$$
\begin{aligned}
\Phi(\alpha(t, 0))|X(t)|= & \int_{0}^{t} \operatorname{sgn}(X(s)) \Phi(\alpha(s, 0)) d X(s) \\
& +\int_{0}^{\alpha(t, 0)} \Phi(z) d z .
\end{aligned}
$$

При этом

$$
\int_{0}^{\alpha(t, 0)} \Phi(z) d z=-\min _{0 \leqslant \tau \leqslant t} \min \left(\int_{0}^{\tau} \operatorname{sgn}(X(s)) \Phi(\alpha(s, 0)) d X(s), 0\right) .
$$

Д ок а з а те л с т в о. Умножая обе части формулы (34) на функщию $\psi(x)$ и интегрируя по $x \in[0,+\infty)$, в силу упомянутой выше 
«стохастической теоремы Фубини», получим: с вероятностью 1

$$
\begin{aligned}
\frac{1}{2} \int_{0}^{t} \Phi(\alpha(s, u)) \alpha(d s, u)= & \Phi(\alpha(t, u))(X(t)-u)^{+}-(-u)^{+} \Phi(0) \\
& -\int_{0}^{t} \mathbb{I}\{X(s)>u\} \Phi(\alpha(s, u)) d X(s)
\end{aligned}
$$

Полагая в последнем равенстве $u=0$, приходим к соотнопению

$$
\begin{aligned}
\Phi(\alpha(t, 0))(X(t))^{+}= & \int_{0}^{t} \mathbb{I}\{X(s)>0\} \Phi(\alpha(s, 0)) d X(s) \\
& +\frac{1}{2} \int_{0}^{t} \Phi(\alpha(s, 0)) \alpha(d s, 0)
\end{aligned}
$$

Нетрудно видеть, что процесс $(-X(s)), s \geqslant 0$, имеет локальное время в точке $u=-x$, равное $\alpha(t, x)$. Поэтому для этого процесса равенство (42) примет вид

$$
\begin{aligned}
\Phi(\alpha(t, 0))(-X(t))^{+}= & -\int_{0}^{t} \mathbb{I}\{X(s)<0\} \Phi(\alpha(s, 0)) d X(s) \\
& +\frac{1}{2} \int_{0}^{t} \Phi(\alpha(s, 0)) \alpha(d s, 0)
\end{aligned}
$$

Складывая данное равенство с равенством (42), получим

$$
\begin{aligned}
\Phi(\alpha(t, 0))|X(t)|= & \int_{0}^{t} \operatorname{sgn}(X(s)) \Phi(\alpha(s, 0)) d X(s) \\
& +\int_{0}^{t} \Phi(\alpha(s, 0)) \alpha(d s, 0)
\end{aligned}
$$

Поскольку в силу следствия к лемме 1

$$
\int_{0}^{t} \Phi(\alpha(s, 0)) \alpha(d s, 0)=\int_{0}^{\alpha(t, 0)} \Phi(z) d z
$$

то соотнотение (40) вытекает из (43).

Положим

$$
h(t)=\int_{0}^{\alpha(t, 0)} \Phi(z) d z, \quad g(t)=\Phi(\alpha(t, 0))|X(t)|
$$

и покажем, что в этих обозначениях (при $x=0$ ) с вероятностью 1 справедлива лемма 4.2 из монографии [2]. Для этого достаточно убедиться, что функция $h(t)$ возрастает только на множестве $\{t: g(t)=0\}$. Имеем:

$$
\begin{gathered}
\int_{0}^{t} \mathbb{I}\{g(s)=0\} d h(s)=\int_{0}^{t} \mathbb{I}\{\Phi(\alpha(s, 0))|X(s)|=0\} \Phi(\alpha(s, 0)) \alpha(d s, 0) \\
=\int_{0}^{t} \mathbb{I}\{X(s)=0\} \Phi(\alpha(s, 0)) \alpha(d s, 0)=h(t) \quad \text { п.н., }
\end{gathered}
$$


поскольку $\Phi(z)>0$ при $z \geqslant 0$. Следовательно (см. $[2$, с. 127]), в силу следствия к лемме 1 получим соотношение (41).

Найдем аналоги формул (39), (34), (40) и (41), соответствуюшие процессу $\xi(t)$.

Положим $B_{\tau}(t)=\int_{0}^{t} \operatorname{sgn}(X(\tau-s)-X(\tau)) * d X(s)$, где интеграл в правой части есть обратный стохастический интеграл по процессу $X(s)$ (некоторые сведения о таких интегралах приводятся, например, в $[6$, c. $36-38])$.

Предложение 6. Для любого $\tau$ с вероятностью 1

$$
\xi(\tau)=|X(\tau)|-B_{\tau}(\tau) ; \quad \xi(\tau)=-\min _{0 \leqslant s \leqslant \tau} B_{\tau}(s)
$$

Д о к а з а т е л в с т в о. При каждом $\tau$ рассмотрим вспомогательный процесс $Y_{\tau}(s)=X(\tau-s)-X(\tau), s \in[0, \tau]$, который также является процессом броуновского движения. Обозначим через $\alpha_{\tau}(p, y), p \in[0, \tau]$, $y \in \mathbf{R}$, локальное время для пропесса $Y_{\tau}(s)$, точнее, его вариант, с вероятностью 1 непрерывный по переменным $(p, y)$.

Выясним связь между локальным временем $\alpha_{\tau}(p, y)$ нового броуновского движения $Y_{\tau}(s)$ и локальным временем $\alpha(t, y)$ исходного процесса $X(s)$. Имеем: если $p \in[0, \tau]$, то

$$
\begin{aligned}
\int_{0}^{p} \mathbb{I}\left\{Y_{\tau}(s) \leqslant x\right\} d s & =\int_{\tau-p}^{\tau} \mathbb{I}\{X(t)<x+X(\tau)\} d t \\
& =\int_{-\infty}^{x+X(\tau)}[\alpha(\tau, y)-\alpha(\tau-p, y)] d y \quad \text { п.н. }
\end{aligned}
$$

В силу непрерывности локальных времен $\alpha(t, y)$ и $\alpha_{\tau}(t, y)$ можно считать, что для любых $\tau \in[0,1]$ п.н. при всех $p \in[0, \tau], y \in \mathbf{R}$

$$
\alpha_{\tau}(p, y)=\alpha(\tau, y+X(\tau))-\alpha(\tau-t, y+X(\tau)) .
$$

В частности, при $p=\tau, y=0$

$$
\xi(\tau)=\alpha_{\tau}(\tau, 0) \quad \text { п.н. }
$$

Положив в соотношении (39) $Z(t)=Y_{\tau}(t), t \in[0, \tau]$, получим $\left|Y_{\tau}(t)\right|=$ $B_{\tau}(t)+\alpha_{\tau}(t, 0)$ п.н.; в частности, при $t=\tau$, ввиду равенства $(45)$, получим формулу (44).

3 а м е ч а н и е. В силу предложения 3 мы можем, во-первых, найти совместное распределение $\mathbf{P}\left\{X(s) \leqslant x, B_{s}(s) \leqslant y\right\}$ слагаемых, входящих в равенство (44), а, во-вторых, найти локальное время для процесса $B_{\tau}(\tau), \tau \in[0,1]$.

Действительно, полагая в равенстве $(31) f(x)=|x|$, в силу равенст- 
ва (44) имеем:

$$
\begin{aligned}
& \mathbf{P}\left\{X(s) \leqslant x, B_{s}(s) \leqslant y\right\} \\
& \quad=\frac{\partial}{\partial s} \int_{-\infty}^{x} \int_{-\infty}^{y} \mathbf{P}\{\alpha(s, u)>z+|u|\} \mathbb{I}\{|u|>z\} d u d z .
\end{aligned}
$$

Наконещ, в силу предложения 3 , функция $\xi(s)-f(X(s))$, где $f(x)=|x|$, обладает локальным временем $\alpha_{f}(t, x)$; с другой стороны, $\xi(s)-|X(s)|=$ $-B_{s}(s)$ п.н.

Положим

$$
J(\tau, x)=\int_{0}^{\tau} \mathbb{I}\{\xi(s)-\alpha(\tau-s, X(\tau)) \leqslant x, X(\tau-s)-X(\tau)>0\} * d X(s) .
$$

Предложение 7. При любом $\tau \in[0,1]$ существует версия стохастического интеграла $J(\tau, x)$ такая, что для любого $x>0$

$$
\frac{1}{2} \min (x, \xi(\tau))=\mathbb{I}\{\xi(\tau) \leqslant x\}(-X(\tau))^{+}-J(\tau, x) \quad \text { n.н. }
$$

Д о к а з а т е л с с в о. Рассмотрим при каждом фиксированном $\tau \in(0,1]$ случайный процесс $Y_{\tau}(s), s \in[0, \tau]$, определенный выше. Применительно к этому процессу и его локальному времени равенство (34) при $t=\tau$ и $u=0$ в силу соотношения (45) имеет вид: для всех $x>0$

$$
\frac{1}{2} \min (x, \xi(\tau))=\mathbb{I}\{\xi(\tau) \leqslant x\}^{\prime}(-X(\tau))^{+}-J_{0}(\tau, x)
$$

где $J_{0}(\tau, x)=\int_{0}^{\tau} \mathbb{I}\left\{\alpha_{\tau}(s, 0) \leqslant x, Y_{\tau}(s)>0\right\} d Y_{\tau}(s)$. Согласно определению обратного стохастического интеграла, интеграл $J_{0}(\tau, x)$ в равенстве $(47)$ можно записать как обратный стохастический интегралі $J(\tau, x)$ по процессу $X(s)$; следовательно $J(\tau, x)=J_{0}(\tau, x)$ п.н.

3 а м е ч а н и я. 1. Справедливо равенство

$$
\left(J(\tau, x)-\frac{x}{2}\right) \mathbb{I}\{\xi(\tau)>x\}=0 \quad \text { п.н. }
$$

2. Мы можем получить более общую, чем равенство (46), формулу стохастического исчисления, используя подход, предложенный в следствии 3 к теореме 9 .

Умножая тождество (46) на функцию $f(x)$, абсолютная величина которой суммируема, и интегрируя полученное тождество по переменной $x \in[0,+\infty)$, в силу упомянутой выше «стохастической теоремы Фубини» имеем:

$$
\frac{1}{2} \int_{0}^{\xi(\tau)} \int_{y}^{+\infty} f(x) d x d y=\int_{\xi(\tau)}^{+\infty} f(x) d x(-X(\tau))^{+}
$$




$$
\begin{aligned}
& -\int_{0}^{\tau}\left[\int_{\xi(\tau)-\alpha(\tau-s, X(\tau))}^{+\infty} f(x) d x\right] \\
& \times \mathbb{I}\{X(\tau-s)>X(\tau)\} * d X(s)
\end{aligned}
$$

3. Предположим дополнительно, что функция $f(x)$ в соотношении (48) такова, что $f(x)>0$ при всех $x$. Тогда предложение 3 позволяет найти локальное время для процесса, определяемого стохастическим интегралом, входящим в равенство (48). Его можно найти, рассмотрев прощесс $\eta(s)=g_{1}(\xi(s))-g_{2}(\xi(s)) \varphi(X(s))$ c

$$
g_{1}(x)=\frac{1}{2} \int_{0}^{x} \int_{y}^{+\infty} f(z) d z d y, \quad g_{2}(x)=\int_{x}^{+\infty} f(y) d y, \quad \varphi(x)=(-x)^{+} .
$$

4. В предположениях, сформулированных в замечании 3, мы можем найти совместное распределение обратного стохастического интеграла, входящего в равенство (48), и исходного процесса $X(s)$.

Действительно, согласно предложению 3 , процесс $\eta(s)=g_{1}(\xi(s))-$ $g_{2}(\xi(s)) \varphi(X(s))$ с функциями $g_{1}(\cdot), g_{2}(\cdot)$ и $\varphi(\cdot)$, определенными в замечании 3 , обладает локальным временем $\beta_{U}(t, y)$. Точнее,

$$
\int_{0}^{t} \mathbb{I}\{\eta(s) \leqslant x, X(s) \in U\} d s=\int_{-\infty}^{x} \beta_{U}(t, y) d y \quad \text { п.н. }
$$

Взяв от обеих частей тождества математическое ожидание и учитывая тот факт (см. предложение 3 ), что для вычисления $\mathbf{E} \beta_{U}(t, y)$ необходимо знать лишь известное распределение $\mathrm{P}\{\alpha(t, u)>x\}$, получим

$$
\mathbf{P}\{\eta(t) \leqslant x, X(t) \in U\}=\frac{\partial}{\partial t} \int_{-\infty}^{x} \mathbf{E} \beta_{U}(t, y) d y
$$

5. Методика доказательств предложений 6 и 7 позволяет очевидным образом перенести равенства (40) и (41) на процесс $\xi(t)$ : с вероятностью 1

$$
\begin{aligned}
& \Phi(\xi(t))|X(t)|= \int_{0}^{t} \operatorname{sgn}(X(\tau-s)-X(\tau)) \\
& \quad \times \Phi(\xi(\tau)-\alpha(\tau-s, X(\tau))) * d X(s)+\int_{0}^{\xi(t)} \Phi(z) d z \\
& \int_{0}^{\xi(t)} \Phi(z) d z=-\min _{o \leqslant \tau \leqslant t} \min \left(\int_{0}^{\tau} \operatorname{sgn}(X(\tau-s)-X(\tau))\right. \\
&\quad \times \Phi(\xi(\tau)-\alpha(\tau-s, X(\tau))) * d X(s), 0) .
\end{aligned}
$$

Автор глубоко признателен М. Г. Шуру, неоднократные беседы с которым стимулировали работу над статьей. 


\section{СПИСОК ЛИТЕРАТУРЫ}

1. Бородия A. H. Броуновское локальное время. - Успехи матем. наук, 1989, т. 44, B. 2 , c. $7-48$.

2. Ватаяабе C., Икеда $H$. Стохастические дифференциальные уравнения и диффузионные процессы. М.: Наука, 1986, 448 с.

3. Королюх В. С., Портенко Н. И., Скороход А. В., Турбин А. Ф. Справочник по теории вероятностей и математической статистике. М.: Наука, 1985, 640 с.

4. Насыров Ф. С. Об ортогональном разложении локальных времен. Уфа, 1985, 25 с. Деп. в ВИНИТИ 23.05.85, № 3593-85.

5. Насыров Ф. С. О лохальных временах для функцй и случайных процессов. I Теория вероятн. и ее примен., 1995, в. 40, в. 4, с. 798-812.

6. Розовский $Б$. JI. Эволюционные стохастические системы. М.: Наука, 1983, 208 с.

7. Barlow M. T. Continuity of local times for Levy processes. - Z. Wahrscheinlichkeitstheor. verw. Geb., 1985, B. 29, H. 1, S. 23-35.

8. Berman $S$. M. Gaussian sample functions: uniform dimension and Hölder conditions nowhere. - Nagoya Math. J., 1972, v. 46, p. 63-86.

9. Berman $S . M$. Local nondeterminism and local times of Gaussian processes. - Indiana Univ. Math. J., 1973, v. 23, № 1, p. 69-94.

10. Berman S. M. Local times of stochastic processes with positive definite bivariate densities. - Stoch. Process. Appl., 1982, v. 12, № 1, p. 1-26.

11. Berman S. M. Joint continuity of the local times of Markov processes. - Z. Wahrscheinlichkeitstheor. verw. Geb., 1985, B. 69, H. 1, S. 37-46.

12. Geman D. A note on the continuity of local times. - Proc. Amer. Math. Soc., 1976, v. 57 , № 2 , p. $321-326$.

13. Geman D., Horowitz J. Occupation densities. - Ann. Probab., 1980, v. 8, № 1, p. 1-67. 